ALPHA No 29 Diciembre 2009 (303-314)

ISSN 0716-4254

http://alpha.ulagos.cl

\title{
DOS RELATOS DEL EXILIO CATALÁN EN CUBA: TERESA PÀMIES Y JOSEP MARIA POBLET
}

Núria Sabaté Llobera*

Es conocido que la Guerra Civil española y sus secuelas políticas forzaron a muchos escritores a transitar los pasos del exilio. Después de cruzar los Pirineos, una de las alternativas a los campos de concentración franceses la proporcionó el Servicio de Evacuación para Refugiados Españoles (S.E.R.E.) que ofrecía la posibilidad de distribución de los exiliados en diferentes países del continente americano. Aunque Cuba no representaba el destino final de tal viaje, sí se encontraba entre las paradas obligatorias del recorrido que en muchos casos terminaría en México, gracias a la generosa política de acogimiento de refugiados del presidente Lázaro Cárdenas. ${ }^{1}$ Entre los exiliados que se ampararon en el acuerdo mexicano se encuentran los escritores Teresa Pàmies y Josep Maria Poblet quienes, en espera de documentos para acceder a México, pasaron varios meses en Cuba.

Si los exiliados españoles de lengua castellana encontraban, al llegar a Hispanoamérica, un contexto lingüístico muy similar al que habían dejado atrás, el exilio catalán "en todas partes se halló sin contexto alguno" (Espadaler 75). Una forma de superar esta descontextualización para muchos catalanes exiliados fue, precisamente, escribir en su lengua materna. Esto dio lugar a una gran producción de literatura catalana en Hispanoamérica que, en muchos casos, podría calificarse de temática híbrida, por cuanto a las cuestiones relacionadas con el exilio y a la nostalgia por Cataluña se les sumaron cuestiones latinoamericanas. Agustí Bartra autor de Quetzalcóatl, Ramón Vinyes (el sabio catalán de Cien Años de Soledad) quien publicó Entre sambes i bananes o Avel.lí Artís Gener con Paraules dópton el Vell, en la que Europa es conquistada por los Aztecas, son algunos ejemplos de esta literatura catalana del exilio que ha empezado a ser estudiada a raíz del desarrollo de los estudios transatlánticos y en la cual el nivel socio-político puede relacionarse con la recuperación de la memoria histórica (quizás en este caso, incluso, sería apropiada la calificación de memoria literaria).

1 Según la bibliografía encontrada, el exilio catalán en México parece haber sido el más estudiado hasta ahora. Cfr. Sebastiaan Faber. Exile and Cultural Hegemony. Spanish Intelectuals in Mexico, 1939-1975 (2002); Dolores Pla Brugat, María Magdalena Ordóñez y Teresa Férriz Roure. El exilio catalán en México. Notas para su estudio (1997). 


\section{Núria Sabaté Llobera}

Propongo, aquí, el análisis del capítulo dos de Gent del meu exili: inoblidables (Gente de mi exilio: inolvidables, 1975) de Teresa Pàmies y el tercer capítulo de Records vells i històries noves (Recuerdos viejos e historias nuevas, 1941) de Josep María Poblet, dos relatos del temprano exilio que se desarrollan en suelo cubano, en tanto Cuba sirve como espacio de libertad para expresar las ideas prohibidas en la Península por el franquismo. El transitar de los protagonistas por los espacios cubanos permite entender (y funciona de acorde a) sus ideologías como catalanes y republicanos.

Hay que destacar que tanto para Pàmies (Balaguer, 1919) como para Poblet (Montblanc, 1897; Barcelona, 1980) la escritura de exilio como recurso autobiográfico tiene un origen distinto. Para Pàmies, la ficción autobiográfica empieza al terminar su exilio. Importante en el mundo periodístico, Teresa Pàmies no publica su primera novela sino hasta 1970, encontrándose en proceso de lo que Mario Benedetti llama el desexilio, el retorno al lugar que una persona se vio obligada a abandonar. Mercè Picornell nos habla de los motivos que subyacen en Pàmies para empezar a escribir en ese momento de su vida y nos dice que "esdevè escriptora amb la intenció única de donar testimoni de la seva vivència” (se convierte en escritora con la única intención de testimoniar sus vivencias, 11). El retorno de Pàmies a Cataluña interrumpe un itinerario geográfico a partir del cual se inicia su trayectoria literaria.

En cuanto a Josep Maria Poblet, sus objetivos como autor aparecen explícitamente escritos en el prólogo de Records vells i históries noves donde anuncia que no era un gran aficionado a la escritura antes de la imposición franquista que obligaba a utilizar el castellano en Cataluña y prohibía públicamente el uso del catalán

Aquest llibre es pensat i escrit en català, durant la meva permanència en les càlides terres de Cuba, ens hauria plagut publicarlo a Barcelona. L' actual xim-xim tràgic de la hispanidad en marcha, que de fa temps ha posat els seus dominis a casa nostra, privant als catalans d' això tan sagrat com es el lliure exercici de la seva llengua, ens obliga a fer-li veure la llum en aquest Mèxic generós que (...) ens ha obert amorosament els braços (11-12). ${ }^{2}$

\footnotetext{
${ }^{2}$ Este libro está pensado y escrito en catalán, durante mi permanencia en las cálidas tierras de Cuba, me habría gustado publicarlo en Barcelona. El actual ritmo trágico de la hispanidad en marcha, que desde hace tiempo se ha aposentado en nuestra tierra, privando a los catalanes de eso tan sagrado como es el libre ejercicio de su lengua, me obliga a hacerle ver la luz en este México generoso que (...) nos ha abierto cariñosamente los brazos”. Josep Maria Poblet. Records vells i históries noves. México: Cultura (1941):11-12. Citaremos por esta edición. Las traducciones son de la autora de estas notas, excepto cuando se indique de otra forma.
} 
Poblet empieza a escribir al principio de su exilio ya que la situación política, tanto como la prohibición lingüística, son la motivación principal para reivindicar el derecho a expresarse en su lengua materna. El testimonio de Poblet se acerca a la demanda político-lingüística del exiliado que escribe desde el presente de los hechos. Por el contrario, la novelística de Pàmies surge de una reflexión acerca de sus vivencias personales, desde la distancia temporal que le suministra el paso de los años.

\section{GENT DEL MEU EXILI: INOBLIDABLES (1975) DE TERESA PÀMIES}

La abundante obra de Teresa Pàmies aparece relacionada con su experiencia como exiliada, mujer y militante política, sus tres señas de identidad. A nivel de género textual se puede hablar de hibridez pues la autora bebe de la ficción tanto como de la autobiografía, la historia y la literatura testimonial. Su trayectoria literaria y sus obras han sido galardonadas con premios como el Josep Pla (1970) o el Premi d'Honor de les Lletres Catalanes (2001), premio que con anterioridad solamente otra mujer (Mercè Rodoreda) ha logrado. A pesar de que sus novelas son de las más leídas entre el público catalán, la crítica le ha dedicado poca atención y, como indica Gina Herrmann, "Pàmies sigue siendo virtualmente desconocida fuera de Cataluña” (2004:7). ${ }^{3}$

En Gent del meu exili: inoblidables, Pàmies rememora su exilio a través de personas y lugares que han marcado su vida en el extranjero. ${ }^{4}$ Aquí me centro en el capítulo II, que tiene lugar en Cuba, donde la protagonista llega en 1940 procedente de Santo Domingo. ${ }^{5}$ La narración se desarrolla en primera persona y relata, a través de abundantes diálogos y descripciones físicas, el encuentro entre una Teresa Pàmies narradora — que como refugiada republicana se encuentra en La Habana buscando trabajo- y Blay, un catalán de mediana edad afincado en Cuba, propietario de un restaurante.

Resulta interesante destacar que la relación de los protagonistas con sus propios recuerdos se desarrolla de una forma distinta en cada uno de ellos

\footnotetext{
${ }^{3}$ Me atrevería a afirmar, más allá de las palabras de Gina Herrmann, que Teresa Pàmies es también desconocida como escritora para gran parte del público catalán. La propia autora afirma que se ha sentido discriminada en muchas ocasiones "per vella o per comunista" (por vieja o por comunista). Ver su artículo "Preguntes adients” (2005).

${ }^{4}$ No me consta la existencia de una traducción al castellano de tal obra aunque el capítulo segundo sí se encuentra traducido en la antología Cuba en la literatura catalana editada por Alex Broch e Isabel Segura Soriano (2002). Las citas de mi análisis se remiten a la traducción propuesta por Marco Tulio Ramírez incluida en la antología mencionada.

${ }^{5}$ Gent del meu exili: inoblidables está integrado por diecisiete capítulos donde la protagonista recuerda personas que conoció en Francia, Eslovenia, Chequia, México y Cuba. La novela presenta una exposición de las circunstancias del exilio a través de la vida de personajes cuyas historias personales marcan a la protagonista.
} 


\section{Núria Sabaté Llobera}

porque el traslado a Cuba no tiene los mismos objetivos: la joven republicana se acuerda del sufrimiento de los últimos meses de guerra, pero concibe el exilio como una posibilidad de ver el mundo y aprovechar ese conocimiento en su lucha política — "buscaba trabajo, pero solo media jornada porque necesitaba tiempo para otras cosas"- (43). Aunque su juventud se ve truncada por el conflicto bélico, el exilio no es aceptado todavía por la joven como una realidad permanente sino como algo temporal.

La historia de Blay la descubrimos a través del relato de Pàmiesnarradora. Durante los encuentros en el restaurante, Blay le cuenta la dramática historia de su vida y su llegada de niño a Cuba para trabajar para un tío indiano involucrado en negocios ilegales. Cuando un día el tío aparece asesinado, el sobrino es acusado del homicidio. La familia de Blay en Cataluña se entera del suceso y la madre muere de un ataque al corazón a causa del disgusto. Blay acaba siendo declarado inocente del crimen pero su familia todavía le cree culpable: "ya hacía años que no se escribía con su familia, que aún debía de considerarlo culpable de la muerte de su madre" (49). A pesar de la abundancia en que ahora vive, y que contrasta con la pobreza de la joven refugiada, la historia del catalán es la que aparece marcada de nostalgia por su tierra natal, por su familia "que nunca supo de su inocencia" (48) y por su lengua materna "que no había querido olvidar nunca" (49).

La llegada de la catalana al restaurante Empordà —nombre de la región de origen del propietario - dispara en Blay el recuerdo de su infancia y de su juventud que le sirve para poner en orden un pasado alterado por acontecimientos traumáticos. El hecho de que Blay aparezca siempre sentado tras un mostrador, en un rincón, en la parte alta del restaurante - “(a) mano derecha, casi tocando la escalera, había una tarima con mostrador tras la cual vigilaba un hombre de unos sesenta años que roía un imponente cigarro apagado" (42) - se puede interpretar como un deseo de apartarse de la realidad y como una voluntad de no querer participar activamente en las labores que se llevan a cabo en el Empordà. La aparición y el "Bon dia" [Buenos días], de la protagonista, hacen que el aislamiento del hombre quede interrumpido: "[n]o me contestó pero me miró de reojo sin descuidar la vigilancia del servicio formado por dos camareros negros que chorreaban sudor” (42). La interrupción que sufre el catalán es sentida como una invasión del espacio propio a la que el hombre responde con un escrutinio visual de la recién llegada, lo cual hace que se replantee su ida al Empordà

(d)e pie delante del mostrador-caja, me sentí incómoda y arrepentida de haber llamado a la puerta de mi compatriota malcarado. Tentada estuve de huir escaleras abajo y dejarlo correr, pero me parecía un poco 
Dos relatos del exilio catalán en Cuba: Teresa Pàmies y Josep Maria Poblet

indecente desechar tan fácilmente la posibilidad de un trabajo que me habían indicado mis compañeros cubanos (42-3).

El reconocimiento de su idioma materno, en un momento inesperado, fuerza a Blay a apartarse de la enajenación en que vive y lo traslada al momento presente, a partir del cual empieza a rememorar un pasado de angustia que ha reprimido hasta entonces.

Los espacios sirven para poner de manifiesto el sentimiento de estancamiento en la vida del catalán, pues a lo largo del relato aparece siempre encerrado en el comedor o en la cocina. Por contraposición, la refugiada, luego de pasar unos minutos en el restaurante, siente la necesidad de salir a tomar aire fresco

Me senté en un banco del paseo y de cara al mar me preguntaba qué clase de persona sería aquel catalán. [...] Pensaba en todo esto sentada en un banco frente al Caribe, sintiendo, tal vez por primera vez en mi flamante exilio, que tropezaría con muchos problemas si no volvíamos pronto a casa (44-45).

Tanto el restaurante — como lugar de reclusión- como el malecón que se abre de cara al mar actúan como espacios que evocan la posibilidad de rearmar un mundo al que los protagonistas puedan pertenecer puesto que el suyo, el propio, se ha visto quebrado ya sea por la voluntad de hacer las Américas o por la guerra.

El relato desafía los roles sociales tradicionales (calle/espacio exteriorhombre, casa/espacio interior-mujer) en que la figura masculina, Blay, pertenece al espacio interior, el cual se contrapone al espacio exterior al que pertenece la refugiada. La catalana existe en y para el espacio público que comparte con sus compañeros de lucha política. Mientras ella se propone reivindicar sus ideales políticos retomando en Cuba la lucha que quedó inacabada por la pérdida de la guerra en España, Blay intenta reconstruir su pasado regresando a la memoria y sin abandonar su restaurante. Gracias al encuentro, los dos compatriotas tienen la oportunidad de reinterpretar no sólo sus vidas personales sino, también, participar en la reelaboración de una identidad colectiva como miembros de un mismo grupo cultural. Esta recreación es sólo posible en la medida en que existe un sentimiento de complicidad entre ambos: una cultura común y el trauma del forzado desarraigo compartido.

El restaurante abre un espacio de posibilidades en que caben y se exteriorizan la amargura, el maltrato y el sufrimiento de Blay, características que, además del origen, comparte con la refugiada. El exilio como práctica compartida hace que el catalán solidarice con la joven. No le ofrece trabajo 


\section{Núria Sabaté Llobera}

porque no puede, pero le recomienda que vaya a ver a "una catalana en el paseo Martí que [l]e podría dar trabajo" (46) y le proporciona un plato de comida mientras lo necesite. La red de contactos entre gente que proviene de la misma región es puesta de manifiesto en distintas ocasiones. Blay le recomienda que se presente ante otra catalana establecida en Cuba, pero son los propios cubanos, amigos de la refugiada, los que deciden que ésa es la mejor opción

Cuando llegué a Cuba, procedente de Santo Domingo, en 1940, se me planteó el problema de buscar trabajo. Alguien me habló de un catalán que tenía un restaurante en la parte vieja del malecón, la más alejada del puerto. Suponían mis amigos cubanos que un catalán no podría negar ayuda a una catalana, y animada por ese argumento, me presenté al Restaurante Empordà (42).

Este encuentro relaciona a los catalanes que llegaron para hacer las Américas - a finales del siglo XIX o principios del XX - con los exiliados políticos llegados en la década de los 30. Ambos grupos comparten la experiencia transatlántica pero no las motivaciones ya que, en el primer caso, se trataba de un viaje exclusivamente económico y, en el segundo, de un viaje por cuestiones políticas. En cuanto a la muchacha, es interesante notar que a ella no le interesa lo de hacer las Américas. El principal objetivo de la catalana no es enriquecerse como los antiguos indianos sino utilizar el espacio cubano para la lucha política. ${ }^{6}$

Es normal, pues, que en el primer encuentro con Blay la refugiada muestre su preocupación hacia las posibles ideas políticas del restaurador

-Tú eres de Lleida...

No fue una pregunta sino una constatación hecha en un tono que no me gustó, como si ser leridana significara un pecado o una tara. No le contesté. Él prosiguió

-Refugiada.

-Sí, sí señor.

-Republicana.

-Naturalmente.

Si era refugiada sólo podía ser republicana ¿No? (43-4).

\footnotetext{
${ }^{6}$ Sebastiaan Faber en Exile and Cultural Hegemony (2002) establece el término desinterés para ilustrar la diferencia entre los refugiados políticos y los antiguos residentes, es decir, las personas que inmigraron por razones económicas. Aunque Faber centra su estudio en los republicanos que llegaron a México, los relatos aquí estudiados ponen de manifiesto una aplicación más amplia del término.
} 
A pesar de la preocupación que, en un primer momento, siente la republicana por las inclinaciones políticas de su compatriota, pronto descubrimos que a Blay la política no le interesa y el ensimismamiento en que vive hace que no sepa nada de sus familiares desde hace tiempo: "[l]e pregunté cómo había seguido él la guerra de España, pero el hombre no sabía casi nada de eso y ya hacía años que no se escribía con su familia” (49).

La aparición de la muchacha y el hecho de compartir la lengua materna hace que Blay simpatice con ella al cabo de unos pocos encuentros. La historia personal y trágica de Blay emerge de su pasado esperando librarse de un tormento que empezó al abandonar Cataluña. Por esto, cuando la protagonista le anuncia que su tiempo en Cuba ha terminado y está por irse a México, la narradora nos deja saber que "me pareció que lo contrariaba porque, según me confesó, hablando conmigo cada día iba recordando la lengua catalana que no había querido olvidar nunca” (49).

El libro de Pàmies pretende ser una reconciliación con el pasado y con sus gentes. En la obra existe un reconocimiento dedicado exclusivamente a personas específicas - tanto conocidas como anónimas - que solidarizaron con los refugiados que, gracias a su inclusión textual, también pasan a formar parte de la memoria del exilio.

\section{RECORDS VELLS I HISTÒRIES NOVES (1941) DE JOSEP MARIA POBLET}

La narrativa de Poblet es, en gran parte, autobiográfica, como la de Pàmies. En ella destacan dos temas principales: la política y el teatro, cuestiones que aparecen entrelazadas en muchas de sus obras, por cuanto el propio autor se dedicó a ambas actividades durante toda su vida.

El exilio de Poblet empieza en Francia, pero a los pocos meses - $-\mathrm{y}$ bajo la amenaza de la II Guerra Mundial- consigue, gracias al Servicio de Emigración para Republicanos Españoles (S.E.R.E.), el financiamiento necesario para un visado y un billete a Cuba. Pasa unos pocos meses en el Caribe y cinco años en Ciudad de México donde adquiere la ciudadanía mexicana. Después de la experiencia americana recorre varios países del mundo y vive temporalmente en Francia, Andorra e Italia, hasta que en 1948 se acoge a la amnistía y logra restablecerse, después de casi 10 años de exilio, en Cataluña. ${ }^{7}$ Josep Maria Poblet muere en Barcelona en 1980.

El capítulo que analizamos a continuación forma parte de Records vells $i$ històries noves, escrita en Cuba durante la estancia del autor en la isla

\footnotetext{
${ }^{7}$ La información biográfica referente a la vida de Josep Maria Poblet ha sido tomada de su libro autobiográfico Memòries d’un rodamón (1976).
} 


\section{Núria Sabaté Llobera}

aunque publicada en México en 1941. ${ }^{8}$ He seleccionado el capítulo III titulado Can Mero, nombre dado a una pensión-restaurante de La Habana que es regentada por un matrimonio catalán llegado a la isla justo después de la independencia cubana. El texto describe detalladamente los quehaceres de los empleados de Can Mero y la interacción de estos con los inquilinos. Poblet utiliza un lenguaje rico que ejemplifica la mezcla que tiene lugar en la isla donde los diferentes personajes (cubanos — blancos y negros - chinos y catalanes) interactúan cómodamente intercambiando y mezclando rasgos de sus propias culturas. El énfasis de Poblet concierne al grupo catalán, dominante en el recinto y con el que se identifica el narrador. El relato empieza con una descripción analógica de Can Mero, que le sirve al lector para situarse en el contexto catalán-cubano de La Habana en los años 40

[f]ins fa poc a l'Havana existien dues societats catalanes; el "Centre" i "La Beneficència”. A l’acabarse [sic] la guerra d’ Espanya se n’hi afegí una de nova, coneguda per 'Can Mero' [...] on molts dies, hi trobaveu més partidaris del llomillo amb mongetes que a qualsevulla altra de les dues entitats. ${ }^{9}$

El restaurante funciona como espacio de interacción para la comunidad catalana. Se trata de un recinto cerrado que posibilita, como el Empordà en el relato de Pàmies, la reconstrucción del mundo roto por el exilio.

El uso de la lengua catalana y la voluntad de mantenerla en el exilio es una de las características comunes que comparten los catalanes que han abandonado su lugar de origen. Si en Pàmies las observaciones al "pobre catalán” que usa el ampurdanés son frecuentes, no resultan menos las menciones a tal tópico en los capítulos de Poblet. El uso de la lengua y las costumbres que llevan a cabo los catalanes establecidos en el Caribe es percibido por el narrador como un símbolo de resistencia del que quiere ser activamente partícipe, de ahí las descripciones detalladas de la atmósfera de la pensión y de sus trabajadores

\footnotetext{
${ }^{8}$ La obra consta de cinco partes: una introducción y cuatro capítulos. El primer capítulo de Records vells... habla de los últimos días de Poblet como funcionario en Barcelona al final de la Guerra Civil. Los tres últimos capítulos están dedicados a su estancia cubana y se centran en sus actividades teatrales, su relación con los catalanes de Cuba, y la divulgación de las tradiciones de los afro-cubanos a través de sus observaciones personales.

9 "hasta hace poco en La Habana existían dos sociedades catalanas; el "Centre" y "La Beneficiència”. Al acabarse la guerra de España se añadió una nueva, conocida como "Can Mero" [...]en donde muchos días, se encontraban más partidarios del lomo con judías que en cualquier otra de las dos entidades” (101). Records vells i históries noves. México: Cultura, 1941. Citaremos por esta edición.
} 
“Ñico era el 'valet de chambre’ de la pensió. (...) Parlava anglès, deia algunes paraules en la llengua de Dumas i es feia entendre en català" (116-17).

"Les festes familiars o patriòtiques es celebraven al menjador dit 'dels catalans'...” (126). ${ }^{10}$

Es importante notar que los protagonistas de Can Mero se expresan en una mezcla de catalán y cubano. El matrimonio formado por "la senyora Teresina” y “en Mero” hace más de treinta años que vive en Cuba, lugar al que se trasladaron para hacer las Américas a principios de siglo XX, tal como Blay, el protagonista del relato de Pàmies. Teresina y Mero han mantenido lo que más les gusta de su cultura y han adoptado lo que más les ha interesado de la cubana pasando a ser ejemplos de la transculturación teorizada por el antropólogo Fernando Ortiz (2002). Esta relación cultural se observa, sobre todo, a través del bilingüismo de Teresina y de Mero en cuyas frases aparecen intercaladas palabras y expresiones de los dos idiomas

Bueno prou i no comencem. Que hay días que las mujeres us lleveu de punta i a fe de Déu que uno no sabe por donde cojeros [sic] ["Bueno basta y no empecemos. Que hay días en que las mujeres os levantáis del lado izquierdo y a fe de Dios que uno no sabe por donde cogeros”] (109).

El uso de la lengua castellana no es visto, en este caso, como una lengua de imposición —-contrariamente a lo que sucede en Cataluña en ese momento- sino como una opción que permite el enriquecimiento cultural y lingüístico.

Si en el relato de Pàmies el restaurante abría un espacio para el recuerdo y la amargura de Blay, la historia de Poblet nos remite a un espacio en el cual la nostalgia del emigrante y del exiliado no tiene cabida

posats sota aquell recer, les hores transcorrien lleugeres. Nomes en obrir els ulls a la realitat, era quan ens veiem lluny de la terra. Per tal de no trobar-la a faltar fins els rètols estaven escrits en llengua catalana “Llenceu els papers a la galleda. Mercès” (128). ${ }^{11}$

\footnotetext{
${ }^{10}$ Ñico era el "valet de chambre” de la pensión. (...) Hablaba inglés, decía palabras en la lengua de Dumas y se hacía entender en catalán... Las fiestas familiares o patrióticas se celebraban en el comedor llamado “de los catalanes”...

11 "Estando bajo ese techo, las horas pasaban ligeras. Solamente al abrir los ojos a la realidad, era cuando nos veíamos lejos de nuestra tierra. Para no echarla de menos hasta los carteles estaban escritos en lengua catalana "tiren los papeles al cubo. Gracias”.
} 


\section{Núria Sabaté Llobera}

Es significativo notar que Can Mero, como relato de exilio, no centra su reflexión en la condición del sujeto exiliado sino que se enfoca en la experiencia del destierro, en general, y expresa la posibilidad de superación de la nostalgia. Los protagonistas de Poblet reproducen un trozo de Cataluña en el centro de La Habana que les permite olvidar que están lejos de su tierra natal. Al sacar a la luz que en partes de Cuba se puede vivir como en Cataluña, Poblet retoma su objetivo de subvertir los efectos franquistas sobre la cultura catalana. Esto se lleva a cabo gracias a recursos como la ironía y el humor que invaden tanto el relato como el espacio físico del restaurante y que ayudan en la ruptura de tópicos divulgados por los franquistas

les festes patriòtiques es celebraven [...] amb banderes del país i la nostra [...]. Advocats, metges, enginyers, aviadors, comerciants, artistes, escriptors, catedràtics, magistrats, funcionaris, navilers i policies n'hi feren cap a dotzenes. Alguns de pas. D’altres, per a romadre a “Cuba, la bella”. Caràcters diferents, edats contraposades... La coincidencia era absoluta en una sola cosa; la manca de disponibilitats econòmiques. La llegenda del dinero de los rojos, una vegada mes, feia figa). ${ }^{12}$

En esta cita se observa una actitud irónica hacia el pensamiento divulgado por la autoridad franquista según el cual los rojos se apoderaron del dinero de las arcas españolas antes de abandonar el país. Poblet desmiente el suceso con la descripción de la situación de precariedad en que se encuentran los rojos en Cuba y se burla del pensamiento de los nacionalistas españoles. La ironía no sólo funciona a través del contraste con la información política sino que aparece ya en la primera línea al hablar de la confraternidad entre catalanes y cubanos. La bandera "del país“ aparece en las fiestas patrióticas junto a "la nuestra” y de esta forma, lo que en realidad tendría que marcar un contraste nacionalista, apela a la fraternidad y al mutuo respeto.

Puesto que la ironía es una estrategia transideológica, usada a nivel político tanto para debilitar como para reforzar opiniones de cariz ya sea conservador o liberal (Hutcheon, 1994:27) en el caso de Poblet recorta las ideas divulgadas por el gobierno franquista - que considera falsas- y, a la vez, refuerza su propia opinión contraria al Régimen. Las cuestiones políticas sirven de punto de partida para la utilización de la ironía que aparece unida a

\footnotetext{
${ }^{12}$ Las fiestas patrióticas se celebraban (...) con banderas del país y la nuestra (...). Abogados, médicos, ingenieros, aviadores, comerciantes, artistas, escritores, catedráticos, magistrados, funcionarios, navieros y policías llegaban a docenas. Algunos de paso. Otros, para quedarse en “Cuba, la bella”. Caracteres diferentes, edades contrapuestas... La coincidencia era absoluta en una única cosa: la falta de disponibilidades económicas. La leyenda del dinero de los rojos, una vez más se desplomaba (126-127).
} 
cuestiones de jerarquía y de subordinación. En el contexto del restaurante aparecen madrileños y andaluces, pero, por primera vez, los catalanes integran el grupo más grande y eso da pie a que la jerarquía cultural que predomina en la Península quede también subvertida

Per acabar de semblar-nos més situats a la península, a la nostra taula sempre acostumava a haver-hi un ciutadà nascut als voltants de La Cibeles o La Giralda, viva representació de les minories oprimides (i) [sic]. !Una vegada o altra havíem de guanyar els catalans! D’allí si que en sortiria un Estatut sense retall de cap mena (128). ${ }^{13}$

El uso de la ironía en esta cita se puede relacionar con la voluntad de alterar el resultado de la realidad social de formar parte de un grupo étnico minoritario. En el caso de Poblet y en el de los refugiados de su relato, además, hay que añadir el resultado por la pérdida de la Guerra Civil. En Can Mero, en particular, y en el espacio de Cuba, en general, se lleva a cabo una reconstrucción de la memoria colectiva catalana. El texto de Poblet se alza como arma de resistencia contra el olvido, el silencio o la censura histórica y lo hace utilizando Cuba como espacio de denuncia.

\section{CONCLUSIÓN}

Tanto el texto de Teresa Pàmies como el de Josep Maria Poblet nos remiten a la reconstrucción de una memoria colectiva formada por los exiliados de la Guerra Civil, en general, y, en particular, el de los refugiados catalanes en Latinoamérica. Pàmies subvierte el rol tradicionalmente secundario de las mujeres que tanto social como políticamente se enmarca dentro de unos logros masculinos. La protagonista es una mujer catalana refugiada y activista política que da cuenta de sus propias vivencias a la vez que rinde homenaje a quienes colaboraron con los exiliados. La narración de Poblet se alza contra los efectos político-culturales que el franquismo tiene para Cataluña y denuncia la posibilidad de resistir a las imposiciones de una España centralista y centralizada.

\footnotetext{
13 Para terminar de parecer más situados en la península, en nuestra mesa siempre acostumbraba a haber un ciudadano nacido en las cercanías de La Cibeles o La Giralda, viva representación de las minorías oprimidas (¿) [sic]. ¡Alguna vez teníamos que ganar los catalanes! De ahí sí que habría salido un Estatuto sin recortes de ningún tipo (128). Poblet se refiere, aquí, al primer estatuto de autonomía de Cataluña aprobado en 1932 y que definía el estado catalán como un estado autónomo dentro de la República española. El estatuto fue anulado al año siguiente cuando la CEDA (Confederación Española de Derechas Autónomas) ganó las elecciones.
} 
Núria Sabaté Llobera

Cuba, como protagonista de fondo, contextualiza estos dos relatos que incorporan América Latina como geografía literaria en la narrativa catalana.

\author{
Centre College* \\ Facultad de Humanidades \\ Departamento de Estudios Hispánicos \\ 600 West Walnut St. Danville, Ky 40422-1394 (EE. UU.) \\ nuria.sabate-llobera@centre.edu
}

\title{
BIBLIOGRAFÍA
}

BENEDETTI, Mario. Desexilio y otras conjeturas. Buenos Aires: Nueva Imagen, 1985.

BROCH, Alex; SEGURA SORIANO, Isabel (ed.). Cuba en la literatura catalana. Barcelona: Insòlit, 2002.

ESPADALER, Antón. "Literatura catalana y exilio", en Revista de Lenguas y Literaturas Catalana, Gallega y Vasca 3 (enero 1993):75-81.

FABER, Sebastiaan. Exile and Cultural Hegemony. Spanish Intellectuals in Mexico, 1939-1975. Nashville: Vanderbilt, 2002.

HERRMANN, Gina. "Catalonia's Teresa Pàmies: A Communist Literary Life", en The Volunteer. Journal of the Veterans of the Abraham Lincoln Brigade XXVI 2 (Junio 2004):7-14.

HUTCHEON, Linda. Irony's Edge. The Theory and Politics of Irony. Routledge: Londres y Nueva York, 1994.

ORTIZ, Fernando. Contrapunteo cubano del tabaco y el azúcar: advertencia de sus contrastes agrarios, económicos, históricos y sociales, su etnografía y su transculturación. Madrid: Cátedra, 2002.

PÀMIES, Teresa. "Preguntes adients", en Avui, 21 de noviembre de 2005.

------- Gent del meu exili: inoblidables. Barcelona: Galba, 1975.

PLA BRUGAT, Dolores et al. El exilio catalán en México. Notas para su estudio. México: Colegio de Jalisco, 1997.

PICORNELL BELENGUER, Mercè. Discursos testimonials en la literatura catalana rencent (Montserrat Roig i Teresa Pàmies). Barcelona: Abadia de Montserrat, 2002.

POBLET, Josep Maria. Memòries d'un rodamón. Barcelona: Pòrtic, 1976.

------- Records vells i històries noves. México: Cultura, 1941.

SEGURA, Isabel. Viatgers catalans al Carib: Cuba. Barcelona: Publicacions de l'Abadia de Montserrat, 1997. 